\title{
Using cervical spine clearance guidelines in a pediatric population: a survey of physician practices and opinions
}

\author{
Emma C. Burns, BSc, MD*; Natalie L. Yanchar, MD, MSc, FRCSC ${ }^{*}$
}

\section{ABSTRACT}

Background: Unlike in adults, there are currently no standardized, validated guidelines to aid practitioners in clearing the pediatric cervical spine (C-spine). Many pediatric centres in Canada have locally produced, adult-modified guidelines, but the extent to which these or other guidelines are used is unknown. Objective: The purpose of this study was to determine if Canadian physicians are using either locally produced or adult C-spine guidelines to clear the $\mathrm{C}$ - spines of patients $<16$ years of age. The study also characterized the common methods used by physicians to clear pediatric $\mathrm{C}$-spine injuries in terms of clinical examination and radiologic imaging.

Methods: A 20-question survey was distributed to 240 Canadian pediatric emergency physicians and trauma team leaders using the Dillman Total Design Method.

Results: The response rate was $68 \%$. The results showed that $61 \%$ of physicians currently use guidelines to assist in the clearance of pediatric $\mathrm{C}$-spines. Of those physicians not using guidelines, $85 \%$ stated that they would use them if they were available. The clinical criteria most often used to clear pediatric C-spines were a normal neurologic examination (97\%) and the absence of C-spine tenderness (95\%), intoxication (94\%), and distracting injuries (87\%).

Conclusions: Guidelines are commonly used by Canadian physicians when clearing the pediatric C-spine, yet few are validated in children. Those most commonly used are locally developed guidelines, the Canadian C-spine guidelines, or National Emergency X-Radiography Utilization Study (NEXUS) low-risk criteria.

\section{RÉSUMÉ}

Contexte: Contrairement à ce qu'il en est pour les adultes, il n'existe actuellement aucune règle normative et validée pour aider les médecins à déterminer quand enlever le collier cervical chez les enfants. Au Canada, bon nombre de centres hospitaliers pour enfants utilisent leurs propres règles pour adultes modifiées, mais l'on ne sait pas dans quelle mesure ces règles ou d'autres sont utilisées.

Objectif: Le but de cette étude était de déterminer si les médecins au Canada utilisaient des règles locales ou d'autres règles pour adultes pour déterminer quand enlever le collier cervical chez les patients de moins de 16 ans. L'étude a également caractérisé les méthodes couramment utilisées (examen clinique et imagerie radiologique) par les médecins pour faire cette détermination.

Méthode: Un sondage à 20 questions a été distribué à 240 médecins d'urgence pédiatrique et chefs d'équipe de traumatologie au Canada selon la méthode de conception globale de Dillman.

Résultats: Le taux de réponse a été de $68 \%$. Les résultats ont montré que $61 \%$ des médecins utilisaient des règles pour aider à déterminer quand enlever le collier cervical chez les enfants. Parmi les médecins qui ne se servaient pas de règles, $85 \%$ ont déclaré qu'ils le feraient si elles étaient disponibles. Les critères cliniques les plus souvent utilisés pour déterminer quand enlever le collier cervical chez les enfants étaient un examen neurologique normal (97\%), I'absence de sensibilité à la colonne cervicale (95\%), aucune évidence d'intoxication du patient (94\%) et l'absence de blessures distrayante (87 \%).

Conclusion: Les médecins au Canada utilisent couramment des règles pour déterminer quand enlever le collier cervical chez les enfants, mais dans la majorité des cas, ces règles ne sont pas validées pour les enfants. Les règles les plus couramment utilisées sont celles élaborées localement, la Règle canadienne concernant la radiographie de la colonne cervicale et les critères de faible risque de l'étude NEXUS (National Emergency X-Radiography Utilization Study).

Keywords: Canadian C-Spine Rule study, Cervical-Spine, guidelines, NEXUS, pediatric trauma, practice variation

From the *Department of Pediatrics, IWK Health Centre, Halifax, NS.

Correspondence to: Dr. Emma C. Burns, Department of Pediatric Emergency Medicine, Children's Hospital of Eastern Ontario, 401 Smyth Road, Ottawa, ON K1H 8C1; ecmburns@yahoo.ca

This article has been peer reviewed.

(c) Canadian Association of Emergency Physicians 
Using clinical practice guidelines to clear the cervical spine (C-spine) of adults presenting with potential spinal and spinal cord injuries is a widely accepted practice. ${ }^{1}$ Since 2000, two sets of guidelines have been validated for use in the adult population: those arising from the National Emergency X-Radiography Utilization Study (NEXUS) low-risk criteria study ${ }^{2}$ and those from the Canadian C-Spine Rule study. ${ }^{3}$ The purpose of these guidelines is to reduce unnecessary imaging, assist in removing collars from patients quickly, and allow for improved flow through the emergency department while ensuring that a spinal cord injury (SCI) is not missed. ${ }^{4}$

There are currently no standard guidelines to direct practitioners in the process of clearing the $\mathrm{C}$-spine in pediatric patients ( $<17$ years of age). The NEXUS criteria have been analyzed in a subset of pediatric patients; however, they have not been validated in the subset under the age of 9.5 Consequently, the applicability of adult-based guidelines in the clinical clearance of the pediatric C-spine is questioned.

A number of barriers may inhibit development and uptake of pediatric C-spine clearance guidelines. Clearance on clinical grounds can be difficult in younger children as they are less able to communicate important symptoms and are easily distracted by pain and fear while being assessed for a traumatic injury. Because of the variation in maturity and cooperation of children, physicians might vary their practice with each patient as opposed to following strict guidelines when assessing adults. ${ }^{6}$ Also, many obstacles may hinder the development of validated pediatric-specific C-spine clearance guidelines. Low rates of injury make systematic research and guideline validation difficult. In North America, patients younger than 15 years account for less than $10 \%$ of all SCIs, with an annual pediatric SCI rate of only 1 in 100,000 compared to 17 in 100,000 in young adults.

Despite such barriers, many Canadian pediatric hospitals have modified adult-based $\mathrm{C}$-spine guidelines to provide their centre with site-specific recommendations; however, their uptake and usability have not been determined. This study aimed to determine how pediatric emergency physicians and trauma team leaders are clearing the C-spine of patients 16 years and younger, specifically focusing on their use of guidelines.

\section{METHODS}

A 20-item survey was developed based on information in the literature and input from local emergency physicians, pediatric surgeons, and neurosurgeons. The questionnaire had three domains: (1) demographics, (2) opinions and use of guidelines in current practice, and (3) use of imaging modalities in evaluating the $\mathrm{C}$-spine in pediatric trauma. Questions directly querying practice patterns, as well as questions exploring management of various clinical scenarios, were used to survey use of imaging. Content validity and reliability were tested by the methods of Waltz and colleagues. ${ }^{8}$ Briefly, five pediatric surgeons and neurosurgeons from across the country rated the content relevance of each question; items with a mean relevance index of less than 0.80 were deleted. A testretest method was used to determine reliability. Ten senior postgraduate trainees in pediatrics completed the survey once and then again a week later. The level of disagreement was determined for each question by computing a kappa coefficient for "yes/no" questions and analyzing intraclass correlation for items with more than one category of response. Those questions showing poor reliability were reworded for clarity.

Web- and paper-based versions of the survey were prepared and distributed to pediatric emergency physicians and pediatric trauma team leaders working in tertiary care hospitals across Canada using the Dillman Total Design Method.9 Survey recipients were identified using the Pediatric Emergency Research Canada (PERC) database or through trauma coordinators at each major centre in Canada. Returned surveys were included in the data analysis if over $90 \%$ of the questions were answered and the respondent had experience in the past year with clearing at least one pediatric C-spine. Responses were analyzed as descriptive statistics with comparison of proportions by chisquare or Fisher exact test as appropriate. All analyses were done using SPSS version 14.0 (SPSS Inc., Chicago, IL). Ethics approval was received from the IWK Health Centre Research Ethics Board.

\section{RESULTS}

Of the 240 physicians contacted, 164 (68\%) responded. Nine were then excluded as they had not cleared a Cspine in the past year or their responses were incomplete. As seen in Table 1, most were subspecialty trained in pediatric emergency medicine, between the ages of 35 and 44 years, with over 10 years of experience. The largest number of respondents were working in emergency departments with a census of 


\begin{tabular}{|c|c|}
\hline Characteristic & $\%$ \\
\hline \multicolumn{2}{|l|}{ Age, yr } \\
\hline 25-34 & 15.5 \\
\hline $35-44$ & 53.5 \\
\hline $45-54$ & 25.2 \\
\hline$>55$ & 5.8 \\
\hline \multicolumn{2}{|l|}{ Physician training } \\
\hline General pediatrics with emergency fellowship & 41.3 \\
\hline General pediatrics & 23.9 \\
\hline Emergency medicine & 10.3 \\
\hline Pediatric critical care & 9.7 \\
\hline Other* & 12.3 \\
\hline Unknown & 2.6 \\
\hline \multicolumn{2}{|l|}{ Years in practice } \\
\hline$<5$ & 24.5 \\
\hline $5-10$ & 28.4 \\
\hline$>10$ & 47.1 \\
\hline \multicolumn{2}{|l|}{ Trauma team leader } \\
\hline Yes & 43.9 \\
\hline No & 53.5 \\
\hline Unknown & 2.6 \\
\hline \multicolumn{2}{|l|}{ Institutional emergency department visits per year } \\
\hline$<15,000$ & 1.9 \\
\hline $15,000-30,000$ & 16.1 \\
\hline $30,000-60,000$ & 49.0 \\
\hline$>60,000$ & 23.2 \\
\hline I don't know/unknown & 9.6 \\
\hline \multicolumn{2}{|l|}{ Number of pediatric C-spines cleared in the last year } \\
\hline $1-9$ & 18.7 \\
\hline $10-19$ & 22.6 \\
\hline $20-29$ & 14.2 \\
\hline$>30$ & 44.5 \\
\hline
\end{tabular}

30,000 to 60,000 patient visits per year and were clearing $>30$ pediatric C-spines per year. Less than half acted as trauma team leaders at their centres.

Sixty-one percent $(n=94)$ of the respondents reported that they use a guideline "almost always" or "most of the time" when clearing the C-spine of patients less than 16 years of age. Of these, $47.1 \%$ used national, published, adult-based guidelines, whereas $41.4 \%$ used locally developed guidelines and $11.5 \%$ used multiple sources (Table 2 and Table 3). Neither years in practice, experience clearing a pediatric C-spine ( $\geq 20$ cleared per year), nor status as a trauma team leader was associated with the use of guidelines. The type of physician training received did influence the use of guidelines. Those trained in primary care (family medicine or pediatrics) were less likely to use guidelines than those trained in emergency medicine or intensive care $(p=0.006)$.

Of the 53 physicians who reportedly "almost never" used a guideline to clear pediatric C-spines, half stated that this was because they were unaware of any guidelines produced at their centres, whereas 31\% felt that there was not enough evidence to support using such guidelines in patients under 16 years of age. Of the physicians who reported that they did not use guidelines, $86 \%$ stated that they would use a protocol if one were made available to them. The 11 physicians who said that they would not use a guideline if it were made available stated the following reasons: not enough research to support the use of a guideline $(n=7)$, preferred to use their own judgment $(n=1)$, clinical protocols are not useful in general $(n=2)$, and it would depend on the guideline $(n=1)$.

Physicians were surveyed as to which criteria they would use to clear a pediatric $\mathrm{C}$-spine without imaging and based on clinical assessment alone. As seen in Table 4, the most common were a normal neurologic examination (97\%), absence of C-spine tenderness (95\%), absence of intoxication (94\%), and lack of distracting injuries (87\%). The criteria used least often were the age of the patient $(23 \%)$, if the patient was ambulatory (23\%), and a history of loss of consciousness (40\%). Similar responses were provided when presented with a clinical scenario of a 4-year-old, restrained male involved in a high-speed frontal motor vehicle collision and asked what factors preclude Cspine clearance based on clinical findings alone (see Table 4).

With regard to imaging of an alert and cooperative child $<16$ years of age, $95 \%$ of respondents reported "almost always" using plain radiographs as the first modality of radiologic assessment, whereas 5\% stated that they use it "most of the time." Respondents listed the reasons why radiography would be supplanted by computed tomography (CT) as the initial imaging modality as the need for a child to go immediately to CT (especially if CT of the head is required), if the child was young, or if the body habitus would result in poor-quality plain films. If already planning CT of the head, only $21 \%$ of respondents would use CT of the C-spine as the first mode of imaging either "almost always" or "most of the time," whereas $79 \%$ stated that they would do so less than half of the time. 


\begin{tabular}{|c|c|}
\hline Survey response & $\begin{array}{l}\text { Percentage of use of guidelines } \\
\text { (NEXUS, CCSR, or locally } \\
\text { developed) }(n=155)\end{array}$ \\
\hline $\begin{array}{l}90-100 \% \text { of the time } \\
\text { (almost always) }\end{array}$ & $67(43.2)$ \\
\hline $\begin{array}{l}>50 \% \text { of the time } \\
\text { (sometimes) }\end{array}$ & $27(17.4)$ \\
\hline $\begin{array}{l}<50 \% \text { of the time } \\
\text { (occasionally) }\end{array}$ & $7(4.5)$ \\
\hline $\begin{array}{l}0-10 \% \text { of the time } \\
\text { (almost never) }\end{array}$ & $53(34.2)$ \\
\hline Unknown & $1(0.6)$ \\
\hline
\end{tabular}

Eighty-seven percent of all respondents were comfortable interpreting plain radiographs of the C-spine without the assistance of a radiologist. Neither years in practice nor status as a trauma team leader was associated with these patterns of imaging selection.

The final scenario of the survey queried what would be done for an alert and cooperative 8-year-old child who continued to have C-spine tenderness in the context of normal three-view plain radiography as their sole imaging. The majority (61\%) would proceed to active flexion and extension views, often qualifying their response by stating that they would do this only if the range of motion of the patient allowed it. Seventeen percent stated that they would leave the patient in a soft collar and schedule follow-up at a later date, whereas $7.7 \%$ would reassess the patient after administering analgesia. CT or magnetic resonance imaging (MRI) of the C-spine would be used by $7.1 \%$ and $4.5 \%$ of respondents, respectively, and $5.8 \%$ would consult either Orthopedics or Neurosurgery. Only $2 \%$ would do no further imaging and schedule no further follow-up.

\begin{tabular}{|c|c|}
\hline Guideline & $\begin{array}{l}\text { Number of physicians using } \\
\text { guideline }(n=87)(\%)\end{array}$ \\
\hline Local hospital guidelines & $36(41.4)$ \\
\hline Canadian C-spine guidelines & $21(24.1)$ \\
\hline NEXUS & $20(23.0)$ \\
\hline Multiple sources* & $10(11.5)$ \\
\hline \multicolumn{2}{|c|}{$\begin{array}{l}\text { NEXUS }=\text { National Emergency X-Radiography Utilization Study. } \\
\text { *Either NEXUS and Canadian C-spine rules or local guidelines plus either NEXUS or } \\
\text { Canadian C-spine rules. }\end{array}$} \\
\hline
\end{tabular}

\section{DISCUSSION}

The use of clinical C-spine clearance guidelines has the potential to lead to a reduction in unnecessary radiologic imaging, to patients spending less time in an uncomfortable C-spine collar, and to earlier discharges from the emergency department. Although the overall rate of SCI in children is much lower than in adults, occurring in only 1 to $2 \%$ of patients with a potential injury, 60 to $80 \%$ of these SCI occur in the C-spine. ${ }^{10,11}$ Emergency physicians are required to assess and manage many patients less than 16 years of age who present with a potential SCI, and the fear of a poor outcome leads to frequent use of plain radiography. ${ }^{5}$ Although the likelihood of injury is low, the consequences are severe.

There are currently no widely accepted guidelines to assist physicians in clearing the $\mathrm{C}$-spine of immobilized children in the emergency department. Viccellio and colleagues found that applying the NEXUS low-risk protocol in pediatric patients to determine the need for radiography resulted in a $100 \%$ sensitivity $(95 \% \mathrm{CI}$ $87.8-100 \%$ ) of detecting a C-spine injury. ${ }^{5}$ However, the number of children less than 9 years of age who had a negative outcome was too small to appropriately validate the use of the NEXUS criteria in that age group. The estimated sample size of 80,000 children required to definitively validate these guidelines in younger patients makes future validation difficult. Despite the lack of clear guidelines, a survey of 1,360 physicians from the American Academy of Pediatrics and the American College of Emergency Physicians found that $63 \%$ of respondents reported that they would clear the C-spine of a 3-year-old comfortably without radiography. ${ }^{6}$

Recognizing the need to provide guidance for physicians assessing potential C-spine injuries in patients less than 16 year of age, 8 of the 13 pediatric tertiary care hospitals in Canada have produced local guidelines (N.L.Y., unpublished data, 2006). This study revealed that of the physician groups sampled, at least half are using either locally modified adult guidelines or the most widely used adult tools (Canadian C-Spine Rules or NEXUS criteria) to clinically clear the C-spines of children of all ages, despite their not being valid for all ages. The study found that physicians use many of the individual criteria in these published guidelines to clinically clear a pediatric C-spine, such as a normal neurologic examination and Glasgow Coma Scale (GCS) score, 


\begin{tabular}{|lcc|}
\hline Table 4. Criteria used for clinical (nonradiologic) & clearance of pediatric C-spines \\
\hline Clinical criterion & $\begin{array}{c}\text { Proportion of use in } \\
\text { multiple choice } \pm 95 \% \mathrm{Cl} \\
(n=155)\end{array}$ & $\begin{array}{c}\text { Proportion of use in } \\
\text { scenario } \pm 95 \% \mathrm{Cl} \\
(n=155)\end{array}$ \\
\hline No focal neurologic deficits/GCS score $<15$ & $96.1 \pm 2.69$ & $97.4 \pm 2.69$ \\
No midline cervical tenderness & $94.8 \pm 3.43$ & $95.5 \pm 3.09$ \\
Patient is not intoxicated & $94.4 \pm 3.74$ & $\mathrm{NA}$ \\
Presence of distracting injuries & $85.8 \pm 5.46$ & $89.0 \pm 4.93$ \\
Patient is noncooperative with clinical & $\mathrm{NA}$ & $81.9 \pm 6.05$ \\
assessment & $66.4 \pm 7.46$ & $66.5 \pm 7.4$ \\
Nondangerous mechanism & $67.1 \pm 7.4$ & $\mathrm{NA}$ \\
Patient is able to actively rotate neck & $58.7 \pm 7.77$ & $\mathrm{NA}$ \\
Patient is verbal & $\mathrm{NA}$ & $40.0 \pm 7.71$ \\
History of loss of consciousness & $23.2 \pm 6.63$ & $\mathrm{NA}$ \\
Patient is ambulatory & $21.9 \pm 6.52$ & $23.2 \pm 6.63$ \\
Age of the patient & \\
\hline GCS $=$ Glasgow Coma Scale; NA = not applicable as this answer was not an option in this question. & \\
\hline
\end{tabular}

absence of C-spine tenderness, a nonintoxicated patient, and lack of distracting injuries (87\%). Less commonly used criteria were dangerous mechanism and patient ambulation prior to ED presentation, which are criteria used in the Canadian C-Spine Rules, were less commonly used criteria. ${ }^{5}$ Interestingly, many physicians reported that the age of the patient was not a useful part of the clinical assessment, although $59 \%$ indicated that the verbal ability of the child would influence their assessment. This suggests that physicians undertaking clinical assessment of pediatric Cspines after trauma may be less concerned with the anatomic differences between younger and older children and therefore are comfortable applying similar criteria to all pediatric ages.

Unlike others, we did not find any correlation between the length of time that a physician has been practicing or his or her role as a trauma team leader and the likelihood of the physician using guidelines. ${ }^{6}$ There was also no significant difference in terms of how often these groups use plain radiography or CT for investigating patients not cleared clinically. We had hypothesized that some physicians might go directly to CT to image the C-spine in those patients also requiring head imaging, yet $79 \%$ of respondents said that they "almost never" or only "occasionally" used CT under these circumstances. It is possible that physicians working only in the intensive care setting who assess more obtunded patients might have answered this question differently. We had also hypothesized that some physicians would be uncomfortable interpreting the $\mathrm{C}$-spine radiographs in children as the anatomy varies from age group to age group and young children can have pseudosubluxation, complicating interpretation. ${ }^{7}$ Yet $87 \%$ stated that they were comfortable without the assistance of a radiologist. Given that $67 \%$ of our surveyed population is trained in pediatrics, it is possible that they are more comfortable interpreting radiographs with varying growth plates, amounts of vertebral translocation, and prevertebral size.

Despite the increased use and availability of MRI, flexion-extension films are still perceived by many to be an important tool in patients with persistent neck pain, as demonstrated in the literature. ${ }^{7,12}$ Sixty-one percent of respondents use active flexion-extension films, many indicating that they would treat their patients with pain control, followed by reassessment, prior to proceeding to these radiographs. The use of flexion-extension films in the pediatric setting is controversial, however, and has been found by others to be of limited utility. ${ }^{13,14}$

\section{LIMITATIONS}

There are a number of limitations to this study. No information is known about the nonresponders, and those who did not reply to the survey might have conflicting opinions. Also, the majority of physicians surveyed are members of the PERC group, indicating an interest in research and continuing education. Therefore, this group might be more homogeneous in terms of knowledge of clinical guidelines than the general practicing population. The majority of the 
respondents are associated with larger, pediatric centres and so are likely more comfortable with clinical difficulties that younger children pose, possibly making them more accepting of guidelines in this population and more comfortable applying them to younger patients. Given that, ultimately, a pediatric C-spine clearance guideline would be used by emergency physicians across Canada, the possibility should be noted that these results are not generalizable to practices in smaller centres. The fact that those trained in primary care were less likely to use guidelines supports this possibility. Referral bias may also affect the study results. As those surveyed practice at larger, tertiary-level, academic, pediatric hospitals, they see many children, often those with the highest acuity. Physicians practicing in this environment may have greater comfort assessing children as per (adult-) validated guidelines, such as the Canadian C-Spine Rules, and therefore be more likely to use them. The respondents to this survey are also all Canadian physicians, who work in a fiscally challenging environment with less litigation than their colleagues in the United States, so they might have a more positive outlook on guidelines. It is similarly possible that US physicians might have a lower threshold for using CT for imaging the C-spines of children either with or without concomitant head injuries.

Finally, physicians surveyed reported only on their beliefs and stated personal practice. Given that reported practice is not always the same as actual practice, it might be that a widely disseminated Cspine clearance guideline would not be used as often as was reported in this survey. Also, although physicians report using guidelines, we have no indication of whether they are using the guidelines properly; they may just include information from the guidelines in their overall decision-making process.

\section{CONCLUSION}

This study indicates that pediatric emergency physicians and trauma team leaders generally support using guidelines to clear the $\mathrm{C}$-spine in patients $<16$ years of age. Despite the fact that the NEXUS criteria, the Canadian C-spine guidelines, and locally produced guidelines have not been validated for children of all ages, there are still many physicians using these tools to assist their clinical practice. This study allows for consideration of the factors that many Canadian pediatric emergency physicians already deem important.

Acknowledgements: The authors would like to thank Steve van Iderstine for his valuable support with survey dissemination, as well as his technical expertise, and Linda Dodds and Colleen O'Connell from the IWK Perinatal Epidemiology Department for their assistance with the statistics. Funding was provided by a Category A grant from the IWK Health Centre.

Competing interests: None declared.

\section{REFERENCES}

1. Brehaut JC, Stiell IG, Graham ID. Will a new clinical decision rule be widely used? The case of the Canadian CSpine rule. Acad Emerg Med 2006;13:413-20.

2. Hoffman JR, Mower WR, Wolfson AB, et al, for the National Emergency X-Radiography Utilization Study Group. Validity of a set of clinical criteria to rule out injury to the cervical spine in patients with blunt trauma. $N$ Engl 7 Med 2000;343:94-9.

3. Stiell IG, Wells GA, Vandemheen KL, et al. The Canadian $\mathrm{C}$-spine rule for radiography in alert and stable trauma patients. FAMA 2001;286:1841-8.

4. Bandiera G, Stiell IG, Wells GA, et al, for the Canadian CSpine and CT Head Study Group. The Canadian C-spine rule performs better than unstructured physician judgement. Ann Emerg Med 2003;42:395-402.

5. Viccellio P, Simon H, Pressman BD, et al. A prospective multicenter study of cervical spine injury in children. Pediatrics 2001;108(2:)e20-6.

6. Omran HA, Dowd M, Knapp JF. Removing the pediatric cervical collar. Arch Pediatr Adolesc Med 2001;155:162-6.

7. Reilly CW. Pediatric spine trauma. 7 Bone foint Surg Am 2007;89A(Suppl 1):98-107.

8. Waltz CF, Strickland OL, Lenz ER. Reliability and validity of criterion-references measures. In: Waltz CF, editor. Measurement in nursing research. Philadelphia: FA Davis Company, 1986. p. 184-208.

9. Dillman D. Mail and telephone surveys: The Total Design Method. New York: Wiley Interscience, 1978.

10. Platzer P, Jaindl M, Thalhammer G, et al. Cervical spine injuries in pediatric patients. 7 Trauma 2007;62:389-94.

11. Kokosa ER, Keller MS, Rallo MC, et al. Characteristics of pediatric cervical spine injuries. 7 Pediatr Surg 2001;36:100-5.

12. Anderson RCE, Scaife ER, Fenton SJ, et al. Cervical spine clearance after trauma in children. 7 Neurosurg 2006; 105(5 Suppl):361-4.

13. Rana AR, Drongowski R, Breckner G, et al. Traumatic cervical spine injuries: characteristics of missed injuries. $\mathcal{F}$ Pediatr Surg 2009;44:151-5.

14. Insko EK, Gracias VH, Gupta R, et al. Utility of flexion and extension radiographs of the cervical spine in the acute evaluation of blunt trauma. 7 Trauma 2002;53:426-9. 